\title{
Lesion distribution characteristics of deep infiltrating endometriosis with ovarian endometrioma: an observational clinical study
}

Hungling Kwok ${ }^{1}$, Hongye Jiang ${ }^{2}$, Tian Li ${ }^{1}$, Huan Yang ${ }^{3}$, Hui Fei ${ }^{1}$, Li Cheng ${ }^{1}$, Shuzhong Yao ${ }^{2}$ and Shuqin Chen ${ }^{2}$

\begin{abstract}
Background: To investigate the characteristics of deep infiltrating endometriosis (DIE) lesion distribution when associated with ovarian endometrioma (OEM).

Methods: The present study analyzed retrospective data obtained by the First Affiliated Hospital of Sun Yat-sen University, between June 2008 to June 2016. A total of 304 patients underwent laparoscopic surgery for complete removal of endometriosis by one experienced surgeon, and histological confirmation of OEM associated with DIE was conducted for each patient. Clinical data were recorded for each patient from medical, operative and pathological reports. Patients were then divided into two groups according to unilateral or bilateral OEM. Patients with unilateral OEM were subsequently divided into two subgroups according to OEM location (left- or right-hand side) and the diameter of the OEM ( $\leq 50$ and $>50 \mathrm{~mm}$ ). The distribution characteristics of DIE lesions were then compared between the groups.

Results: DIE lesions were widely distributed, 30 anatomical sites were involved. Patients with associated unilateral OEM ( $n=184$ patients) had a significantly increased number of DIE lesions when compared with patients with bilateral OEM ( $n=120$ patients; $2.76 \pm 1.52$ vs. $2.33 \pm 1.34 ; P=0.006)$. Compared with bilateral OEM with DIE, there was a higher rate of intestinal $(39.1 \%$ vs. $18.3 \% ; P<0.01)$ and vaginal $(17.4 \%$ vs. $6.7 \% ; P<0.01)$ infiltration by DIE lesions in unilateral OEM with DIE. The mean number of DIE lesions was not significantly correlated with the location or size of the OEM ( $2.83 \pm 1.56$ vs. $2.74 \pm 1.53 ; P=0.678$; and $2.65 \pm 1.42$ vs. $2.80 \pm 1.43 ; P=0.518$, respectively). There was no significant difference between the groups with OEM $\leq 50 \mathrm{~mm}$ and $>50 \mathrm{~mm}$.
\end{abstract}

Conclusion: Lesion distribution characteristics in women diagnosed with histologically proven OEM associated with DIE were frequently multifocal and severe.

Keywords: Ovarian endometrioma, Deep infiltrating endometriosis, Lesion distribution characteristics

\section{Background}

Endometriosis is a common benign diseases of women of childbearing age [1]. According to histology,

\footnotetext{
* Correspondence: chenshuqin1021@163.com

${ }^{2}$ Department of Obstetrics and Gynecology, the First Affiliated Hospital, Sun Yat-sen University, Guangzhou 510000, China

Full list of author information is available at the end of the article
}

endometriosis is characterized into three main types: superficial endometriosis, ovarian endometrioma and deep infiltrating endometriosis (DIE) [2]. DIE is an aggressive form of the disease, penetrating to more than $5 \mathrm{~mm}$ under the peritoneal surface [3].DIE is associated with infertility and a variable degree of pelvic pain. It is a multifocal disease primarily affecting the posterior area, and frequently

(c) The Author(s). 2020 Open Access This article is licensed under a Creative Commons Attribution 4.0 International License, which permits use, sharing, adaptation, distribution and reproduction in any medium or format, as long as you give appropriate credit to the original author(s) and the source, provide a link to the Creative Commons licence, and indicate if changes were made. The images or other third party material in this article are included in the article's Creative Commons licence, unless indicated otherwise in a credit line to the material. If material is not included in the article's Creative Commons licence and your intended use is not permitted by statutory regulation or exceeds the permitted use, you will need to obtain permission directly from the copyright holder. To view a copy of this licence, visit http://creativecommons.org/licenses/by/4.0/. The Creative Commons Public Domain Dedication waiver (http://creativecommons.org/publicdomain/zero/1.0/) applies to the data made available in this article, unless otherwise stated in a credit line to the data. 
involves the uterosacral ligament, uterine rectum pouch and vaginal rectal diaphragm, as well as the bladder, ureter and rectal wall [4].

For patients with multifocal characteristics of DIE, meaningful improvements in clinical symptoms and quality of life are dependent on the radical exeresis of the lesions $[5,6]$. When surgical treatment has been recommended, an accurate diagnosis and knowledge of the precise distribution of the extending lesions are required [4]. However, there are currently no adequately sensitive and specific symptoms nor diagnostic tests for the clinical diagnosis of DIE. The perfect solution would be to utilize a preoperative label for the distribution of DIE to generate a precise map of the DIE lesions.

Ovarian endometrioma (OEM) is the most common type of endometriosis, $~ 50 \%$ of DIE patients are also diagnosed with OEM [7]. Previous studies have demonstrated that associated OEM is a marker for greater DIE severity. Compared with patients without OEM, patients with OEM have more pelvic and intestinal areas involved by DIE $[8,9]$. In case of OEM, severe pelvic pain is significantly associated with DIE [10]. Indeed, Perello et al. [11] created a predictive model to predict DIE in patient with OEM. However, these studies do not refer to the characteristics of the lesion distribution in OEM patients with DIE. Therefore, the present study investigated OEM in order to determine the lesion distribution characteristics of DIE associated with OEM.

\section{Methods}

\section{Patients}

The present retrospective study was approved by the Institutional Review Board of the First Affiliated Hospital of Sun Yat-sen University (Guangzhou, China). All patients provided written informed consent. A total of 304 consecutive patients underwent laparoscopic surgery for complete removal of endometriosis in the First Affiliated Hospital of Sun Yat-sen University, between June 2008 to June 2016; histological confirmation of OEM associated with DIE was conducted for each patient. Histological diagnoses were based on characteristics previously defined in a standard histological description, comprised of a combination of endometrial glands and stroma [12].

The exclusion criteria were as follows: i) laparoscopic surgery converted to open surgery; ii) lack of histological confirmation of endometriosis; iii) previous history of hysterectomy or oophorectomy; and iv) patients with pelvic malignant tumors.

Between June 2008 to June 2016, clinical data were recorded retrospectively for each patient from medical, operative and pathological reports; data were blinded for examination by two of the authors. For each patient, general data were recorded, including age, parity, the diameter and location of the OEM (right or left), and past history of surgical treatment for endometriosis, pain symptoms and other specific symptoms. Pain symptoms included dysmenorrhea, dyspareunia, chronic pelvic pain, and bowel pain. Other specific symptoms including hematochezia, urinary frequency and urgency, and hydro-nephrosis. Histological confirmation was obtained for all endometriotic lesions and a description of the location of the DIE lesions was recorded.

All of the patients underwent extensive preoperative tests, including clinical examinations, transvaginal sonography, urological ultrasound and MRI. Patients were treated surgically due to ineffective conservative treatment or serious complications. All of the operations were performed by an experienced surgeon (Professor Shuzhong Yao). All visible endometriosis lesions, including ovarian endometrioma and DIE lesions, were surgically removed. For patients with adenomyosis, local resection or hysterectomy was performed according to the severity of the disease and bearing requirement. In addition to DIE resection, surgical treatment involves adhesion separation as well as resection and reconstruction of the urinary organs and bowel. When these procedures were employed, urologists and gastroenterologists were necessary. Patients were divided into two groups according to the type OEM: unilateral or bilateral. Patients with unilateral OEM were subsequently divided into subgroups according to the location of the OEM (left- or right-hand side) and the diameter of the OEM $(\leq 50$ and $>50 \mathrm{~mm})$. The distribution characteristics of DIE lesions were then compared between the groups.

\section{Statistical analysis}

Statistical analysis was performed using SPSS version 23.0 for Windows (IBM Corp., Armani, NY, USA). $P<$ 0.05 was considered to indicate a statistically significant difference. Statistical analysis was conducted stepwise after assessing quantitative variable distributions. Statistical studies using parametric tests were only conducted after checking the normal distribution of the studied variables. Correlations between qualitative variables were analyzed by a Pearson's chi-square test. In case of significant reciprocal correlation, the relative risk was calculated with $95 \%$ confidence intervals.

\section{Results}

Patient characteristics are presented in Table 1. A total of 304 patients were included in the present study. The female patients had a mean age of $34.2 \pm 5.8$ (range, 1749) years. All the patients with OEM associated with DIE have different symptoms. According to the different symptoms, they were divided into two types: nonspecific symptoms and specific symptoms. Non-specific symptoms include dysmenorrhea (89.5\%), dyspareunia $(12.8 \%)$ and chronic pelvic pain (4.3\%), while specific 
Table 1 Preoperative characteristics of the study population

\begin{tabular}{ll}
\hline Patients' characteristics $(\mathrm{N}=304)$ & Values ${ }^{\mathrm{a}}$ \\
\hline Age(years) & $34.2 \pm 5.8($ range 17-49) \\
Parity & \\
Para $\geq 1$ & $158(52.0 \%)$ \\
Nulliparous & $146(48.0 \%)$ \\
Infertility & $78(25.7 \%)$ \\
Previous surgery for OEM & $88(28.9 \%)$ \\
Presenting symptoms & \\
No symptoms & $32(10.5 \%)$ \\
Dysmenorrhea & $272(89.5 \%)$ \\
Dyspareunia & $39(12.8 \%)$ \\
Chronic pelvic pain & $17(5.6 \%)$ \\
Rectal tenesmus & $73(24.0 \%)$ \\
Difficulty in defecation & $13(4.3 \%)$ \\
Diarrhea & $6(2.0 \%)$ \\
Defecation pain & $11(3.6 \%)$ \\
Hematochezia & $8(2.6 \%)$ \\
Urinary frequency and urgency & $9(3.0 \%)$ \\
Haematuria & $2(0.7 \%)$ \\
Hydronephrosis & $26(8.6 \%)$ \\
Endometrioma laterality & \\
Left & $98(32.2 \%)$ \\
Numberal & $86(28.3 \%)$ \\
\hline aEM, ovarian endometrioma; DIE, deep infiltrating endometriosis & $120(39.5 \%)$ \\
bifferent symptoms can be associated in the same patient
\end{tabular}

symptoms include intestinal symptoms and urinary symptoms. Intestinal symptoms include rectal tenesmus (24\%), difficulty in defecation (4.3\%), defecation pain (3.6\%), diarrhea (2.0\%) and hematochezia (2.6\%). Urinary symptoms include urinary frequency and urgency (3.0\%), hematuria $(0.7 \%)$ and hydronephrosis $(8.6 \%)$. In the cohort of 304 patients with an associated OEM, the OEM was located on the left-hand side in 98 patients (32.2\%), on the right-hand side in 86 patients $(28.3 \%)$, and was bilateral in 120 patients $(39.5 \%)$. The mean size of the OEM was $55.9 \pm 23.9 \mathrm{~mm}$ (range, $10-150 \mathrm{~mm}$ ).

The anatomical locations of the DIE lesions are presented in Table 2. A total of 788 histologically confirmed DIE lesions were observed in the present study. The mean number of DIE lesions per patient was $2.60 \pm 1.46$ (range, $1-9$ ). DIE lesions were widely distributed; there were 30 anatomical sites recorded in the present study, which were mainly located in the posterior pelvic cavity. A total of 532 lesions were recorded, of which 274 (51.5\%) were located on the
Table 2 The prevalence of anatomical distribution of DIE lesions. ( $\mathrm{n}=304$ patients)

\begin{tabular}{ll}
\hline Main lesion $^{\text {a }}$ & Number of patients $(\%)^{b}$ \\
\hline Uterosacral ligament & $245(80.6)$ \\
Left & 57 \\
Right & 53 \\
Bilateral & 135 \\
Intestine & $93(30.6)$ \\
Ureter & $47(15.5)$ \\
Left & 22 \\
Right & 18 \\
Bilateral & 7 \\
Vaginal & $40(13.2)$ \\
Posterior fornix & $33(10.9)$ \\
Fallopian tube & $29(9.54)$ \\
Left & 12 \\
Right & 4 \\
Bilateral & 13 \\
Recto-vaginal septum & $21(6.91)$ \\
Rectovaginal pouch & $9(2.96)$ \\
Bladder & $4(1.32)$ \\
\hline
\end{tabular}

DIE deep infiltrating endometriosis

${ }^{a}$ According to the location of the lesion recorded during the operation ${ }^{b}$ Number of patients whose lesions histologically proven deep infiltrating endometriosis

left-hand side and 258 (48.5\%) on the right-hand side. The uterosacral ligament represented the most frequent location site with a prevalence of $80.6 \%$, followed by the intestine (30.6\%), ureter $(15.5 \%)$, vagina $(13.2 \%)$, posterior fornix (10.9\%), fallopian tube (9.54\%), vaginal-rectum (6.91\%), rectovaginal pouch $(2.96 \%)$ and bladder (1.32\%). Intestinal endometriosis includes rectum and sigmoid endometriosis. There were also 4 cases of appendiceal endometriosis that were not included in the intestinal endometriosis.

Patients with associated unilateral OEM $(n=184$ patients) had a significantly increased number of DIE lesions when compared with patients with bilateral OEM ( $n=120$ patients; $2.76 \pm 1.52$ vs. $2.33 \pm 1.34 ; P=0.006$; Table 3). Compared with bilateral OEM with DIE, a higher rate of intestinal $(39.1 \%$ vs. $18.3 \% ; P<0.01)$ and vaginal $(17.4 \%$ vs. $6.7 \%$; $\mathrm{P}<0.01)$ infiltration by $\mathrm{DIE}$ lesions was observed in unilateral OEM with DIE. The mean number of DIE lesions was not significantly correlated with the location or size of the OEM $(2.83 \pm 1.56$ vs. $2.74 \pm 1.53, P=0.678 ; 2.65 \pm 1.42$ vs. $2.80 \pm 1.43, P=$ 0.518 , respectively). There were no significant differences between the groups with OEMs $\leq 50 \mathrm{~mm}$ and $>50 \mathrm{~mm}$.

\section{Discussion}

Previous studies have revealed that, for patients with histologically confirmed DIE, an associated ovarian OEM is 
Table 3 Characteristics of deeply infiltrating endometriosis lesion distribution associated ovarian endometrioma

\begin{tabular}{|c|c|c|c|c|c|}
\hline \multirow[t]{3}{*}{ Variables } & \multicolumn{2}{|l|}{ Comparison } & \multirow{3}{*}{$\begin{array}{l}P \text { - } \\
\text { value }\end{array}$} & \multirow[t]{3}{*}{ OR } & \multirow[t]{3}{*}{$95 \% \mathrm{Cl}$} \\
\hline & \multicolumn{2}{|l|}{$\mathrm{OEM}_{\text {laterality }}{ }^{\mathrm{a}}$} & & & \\
\hline & Unilateral $n=184(\%)$ & Bilateral $n=120(\%)$ & & & \\
\hline Number of DIE lesions & $2.76 \pm 1.52$ & $2.33 \pm 1.34$ & $0.006^{*}$ & & \\
\hline Uterosacral ligament & $151(82.1)$ & $94(78.3)$ & 0.421 & 1.266 & $0.712-2.249$ \\
\hline Intestine & $72(39.1)$ & $21(17.5)$ & $0.000^{*}$ & 3.031 & $1.738-5.286$ \\
\hline Vagina & $32(17.4)$ & $8(6.70)$ & $0.007^{*}$ & 2.947 & $1.308-6.641$ \\
\hline Posterior fornix & 23(12.5) & $10(8.3)$ & 0.100 & 1.571 & $0.720-3.431$ \\
\hline Ureter & 29(15.8) & 18(15.0) & 0.858 & 1.060 & $0.560-2.009$ \\
\hline Fallopian tube & 19(10.3) & $10(8.3)$ & 0.563 & 1.267 & $0.568-2.827$ \\
\hline \multirow[t]{3}{*}{ Rectovaginal pouch } & $16(8.7)$ & 14(11.7) & 0.396 & 0.767 & $0.359-1.637$ \\
\hline & \multicolumn{2}{|l|}{ Side of $\mathrm{OEM}^{\mathrm{a}}$} & & & \\
\hline & Left side $n=98(\%)$ & Right side $n=86(\%)$ & & & \\
\hline Number of DIE lesions & $2.83 \pm 1.56$ & $2.74 \pm 1.53$ & 0.678 & & \\
\hline Uterosacral ligament & $81(82.7)$ & $70(81.4)$ & 0.824 & 1.089 & $0.512-2.315$ \\
\hline Intestine & $40(40.8)$ & $32(37.2)$ & 0.617 & 1.164 & $0.642-2.109$ \\
\hline Vagina & 18(18.4) & $15(17.4)$ & 0.870 & 1.065 & $0.500-2.268$ \\
\hline Posterior fornix & 15(15.3) & $8(9.3)$ & 0.219 & 1.762 & $0.078-4.386$ \\
\hline Ureter & 16(16.3) & 13(15.1) & 0.822 & 1.096 & $0.494-2.431$ \\
\hline Fallopian tube & $8(8.2)$ & $11(12.8)$ & 0.303 & 0.606 & $0.232-1.584$ \\
\hline \multirow[t]{3}{*}{ Rectovaginal pouch } & $9(9.2)$ & $7(8.1)$ & 0.802 & 1.141 & $0.406-3.207$ \\
\hline & \multicolumn{2}{|l|}{ Unilateral OEM size ${ }^{a}$} & & & \\
\hline & $\leq 50 \mathrm{~mm} \mathrm{n}=112(\%)$ & $>50 \mathrm{~mm} \mathrm{n}=72(\%)$ & & & \\
\hline Number of DIE lesions & $2.77 \pm 1.4$ & $2.82 \pm 1.4$ & 0.960 & & \\
\hline Uterosacral ligament & $87(77.7)$ & 64(88.9) & 0.053 & 0.435 & $0.184-1.027$ \\
\hline Intestine & $54(48.2)$ & 17(23.6) & $0.001^{*}$ & 3.012 & $1.560-5.817$ \\
\hline Vagina & $25(22.3)$ & $6(8.3)$ & $0.013^{*}$ & 3.161 & $1.226-8.164$ \\
\hline Posterior fornix & $11(9.8)$ & $12(16.7)$ & 0.171 & 0.545 & $0.226-1.311$ \\
\hline Ureter & 15(13.4) & 14(19.4) & 0.272 & 0.641 & $0.289-1.423$ \\
\hline Fallopian tube & 10(8.9) & $9(12.5)$ & 0.437 & 0.686 & $0.264-1.781$ \\
\hline Rectovaginal pouch & $11(9.8)$ & 12(16.7) & 0.171 & 0.545 & $0.226-1.311$ \\
\hline
\end{tabular}

Values are shown as mean \pm standard deviation or $N(\%)$

*Statistically significant

a marker for the severity of the disease [8]. However, the related research on the distribution characteristics of the DIE lesions in patients with OEM is still limited. The present study investigated lesion distribution characteristics in women diagnosed with histologically confirmed OEM and DIE, as this combined diagnosis is often indicative of a more multifocal and severe disease. There was a greater frequency of anatomic DIE lesion sites in patients with unilateral OEM (unilateral OEM size $\leq 50$ $\mathrm{mm}$ ) than in patients with bilateral OEM (unilateral OEM size $\leq 50 \mathrm{~mm}$ ), and DIE lesions were often associated with intestinal and vaginal infiltration.

These data suggested that DIE lesions were widely distributed. There were 30 anatomical sites observed in the present study, which were primarily located in the posterior pelvic cavity, but also in the uterosacral ligaments, intestines, ureter, vagina, posterior fornix and rectovaginal pouch. The distribution of DIE lesions was associated with the flow pattern of peritoneal fluid and the morphology of the pelvic cavity [13]. When compared with the uterosacral ligaments, intestines and vagina, the reduced frequency of deep bladder endometriosis observed can be explained by anatomical location as the lower limit of the vesico-uterine pouch is located well above the lower limit of the Pouch of Douglas [14]. The anatomical differences between the left- and right-hand sides of the hemipelvis are that the sigmoid colon is located on the left-hand side of the hemipelvis, and when 
combined with the left adnexa it forms a barrier to prevent menstrual blood reflux, resulting in an anatomical situation that could promote adhesions and the growth of refluxed endometrial cells on the left-hand side of the pelvic wall [15].

According to the literature, intestinal endometriosis and urinary tract endometriosis account for $\sim 5-12 \%$ and $0.3-12 \%$, respectively, of all women with endometriosis [16-18]. However, in the present study, intestinal endometriosis accounted for $30.9 \%$, and urinary tract endometriosis accounted for $15.2 \%$ of cases, which are greater frequencies than those recorded by previous research. According to the theory of Kondo et al., women presenting with OEM had a stronger association with the presence of DIE lesions and intestinal DIE [19]. There is lack of research on OEM associated with urinary tract endometriosis. The present results indicated that, for patients diagnosed with histologically verified OEM associated with DIE, there was an increased risk of lesions involving the intestine and ureter. Thus, when the clinical examination suggests OEM with DIE, the practitioner should search for severe lesions, especially intestinal and ureteral lesions. Surgery for intestinal endometriosis and urinary endometriosis is difficult, with a number of postoperative complications [20,21]. The literature reports distinguish between major and minor complications. Major complications include anastomotic insufficiencies, intestinal perforation, retovaginal fistulas, severe infections, and bleeding requiring transfusion, which are reported in 7.4\% [22] to 25\% [23]. Minor complications include slight-to-moderate infections, peripheral sensory disturbances, bladder voiding dysfunction, and postoperative urinary obstruction, which are reported in $0.6 \%$ [24] to $57 \%$ [25]. Therefore, full clinical evaluations, as aforementioned, before operating may be very important for OEM patients with DIE.

As mentioned above, intestinal endometriosis refers to the rectum and sigmoid endometriosis. However, there are a special intestinal endometriosis, appendiceal endometriosis, which is considered as an uncommon finding, in the literature its prevalence varies widely [26]. In our study, there are 4 patients with appendiceal endometriosis , are responsible for approximately $4 \%$ of all intestinal lesions. Mabrouk et al. [27] pointed out that appendiceal endometriosis was associated with adenomyosis, large right endometrioma, deep posterior pelvic endometriosis, left deep lateral pelvic endometriosis, and ileocecal involvement. The 4 patients in our study who had appendiceal endometriosis were all combined with adenomyosis, and the diameter of OEM was greater than $5 \mathrm{~cm}$, which was further validated the conclusions of Mabrouk et al.

Patients with simple OEM often have no typical clinical manifestations, and some patients only find the presence of OEM in routine physical. Previous studies have conducted multivariate regression analysis on the relationship between dysmenorrhea and OEM. The results showed that the severity of dysmenorrhea had nothing to do with the existence of OEM. Intestinal endometriosis and deep pelvic invasive endometriosis were the main related factors of dysmenorrhea [28]. Of the 304 patients in our study, 272 (89.5\%) with symptoms of dysmenorrhea, 39 (12.8\%) with dyspareunia, and 17 (5.6\%) with chronic pelvic pain. Dai et al. reported that $61.6 \%$ of patients with OEM but non-DIE had dysmenorrhea, and $10.2 \%$ had severe dysmenorrhea [29]. It is suggested that when patients with OEM have obvious pain symptoms, it should be considered that they may be complicated with DIE.

During the early stages of ureteral endometriosis, the clinical symptoms are not typical, and are easily overlooked during surgery. Some researchers have suggested that urinary tract endometriosis may occur more frequently than it is currently thought to [30, 31]. Raimondo $\mathrm{D}$ et al. observed that ureteral involvement was always associated with endometriosis in other locations in the pelvis. And the most frequent endometriosis associations with ureteral involvement are ovarian lesions [32]. In this case, when there is an associated OEM, ultrasonic examination of the urogenital system is necessary. Ureteral endometriosis should be considered when B mode ultrasound reveals ureter stenosis and hydronephrosis. Hydroureter and hydronephrosis are the severe forms of ureteral endometriosis. In case of hydronephrosis, renal scintigraphy to evaluate renal function should be needed.

Patients with associated unilateral OEM have an increased number of DIE lesions and have a greater risk of lesions involving the intestine and vagina when compared with patients with bilateral OEM. It was hypothesized that a clinical case of bilateral OEM may be more complex than unilateral OEM, and the distribution of the DIE lesions may be more extensive and deeper in patients with larger OEM. However, some researchers maintain that in patients with OEM $\geq 30 \mathrm{~mm}$, OEM size was the most influential contributor to the total number of follicles and oocytes retrieved. OEM results in a reduced response to ovarian stimulation, when compared with the response of the contralateral normal ovary in the same individual [33]. The oppression of the ovary was more obvious in bilateral OEM and the response to ovarian stimulation was markedly reduced. In the clinic, patients with unilateral OEM or an OEM with a diameter of $\leq 50 \mathrm{~mm}$ should not be taken lightly, and should be evaluated for the presence of DIE lesions, especially intestinal DIE.

The aim of the present study was to potentially provide the basis for avoiding underestimation of the extent of the DIE lesions. Misunderstanding the severity of DIE lesions is the main reason why it is difficult to completely remove lesions during surgical treatments. 
Incomplete exeresis of DIE lesions explains the high risk of recurrence; this recurrence is in fact the continued progression of the lesions left behind during previous operations [34, 35]. Repeated surgery is positively associated with increased health care costs and morbidity [36]. Repeated surgery with damage to ovarian reserves is particularly frequent in ovarian endometriosis [37-39]. In addition, the risk of repeated operations is associated with uncertainty regarding surgical outcomes and pain. Therefore, completely excising the DIE lesions during the initial surgery is particularly important.

For the first time, our study analyses the characteristic of DIE lesion distribution in patient with ovarian endometrioma. However, this was a retrospective single center study, results were limited by a lack of random, patient selection, and incomplete data acquisition. The data on the history of previous endometriosis treatment were incomplete, which prevented us from evaluate the effect of previous treatment on the recurrence. A large multicenter prospective trial will be necessary to further assess this lesion distribution characteristic.

\section{Conclusions}

In summary, lesion distribution of DIE associated with OEM was frequently multifocal and severe. For patients with OEM that requires surgical treatment, it cannot be treated only by cystectomy; it should be combined with the patient's clinical symptoms, and be carefully explored during surgery to avoid the omission of the lesions.

\section{Abbreviations}

DIE: Deep Infiltrating Endometriosis; OEM: Ovarian Endometrioma

\section{Acknowledgements}

The authors want to warmly thank staff members from our department of medical record management for their expert assistance with data collection. The authors also thank Dr. Xiao Lin for his help in preparing the statistical analysis.

\section{Authors' contributions}

SC and SY: conceived and designed the study. HK and $\mathrm{HJ}$ : contributed to data analysed and interpreted the data, writing the manuscript. TL and HY: involved in manuscript writing. HF and LC: collected the Data. All the authors approve the final version of the manuscript.

\section{Funding}

This work was supported by the Natural Science Foundation of China (81070472) and the Science and Technology Planning Project of Guangdong Province (2012B031800121).

\section{Availability of data and materials}

The datasets used and/or analyzed during the current study are available from the corresponding author on reasonable request.

\section{Ethics approval and consent to participate}

The study was approved by Ethics Committee of the First Affiliated Hospital of Sun Yat-sen University, and the written consent was obtained from participants.

\section{Consent for publication}

Not applicable.

\section{Competing interests}

The authors declare that they have no competing interest and nothing to disclose.

\section{Author details}

'Department of Obstetrics and Gynecology, the Seventh Affiliated Hospital of Sun Yat-sen University, Shenzhen, China. ${ }^{2}$ Department of Obstetrics and Gynecology, the First Affiliated Hospital, Sun Yat-sen University, Guangzhou 510000, China. ${ }^{3}$ Department of Gynecology, Shenzhen Second People's Hospital/ the First Affiliated Hospital of Shenzhen University Health Science Center, Shenzhen, China.

Received: 19 December 2019 Accepted: 10 May 2020

Published online: 20 May 2020

\section{References}

1. Vercellini $P$, et al. Endometriosis: pathogenesis and treatment. Nat Rev Endocrinol. 2014;10(5):261-75.

2. Cornillie FJ, et al. Deeply infiltrating pelvic endometriosis: histology and clinical significance. Fertil Steril. 1990;53(6):978-83.

3. Koninckx PR, et al. Suggestive evidence that pelvic endometriosis is a progressive disease, whereas deeply infiltrating endometriosis is associated with pelvic pain. Fertil Steril. 1991;55(4):759-65.

4. Chapron C, et al. Anatomical distribution of deeply infiltrating endometriosis: surgical implications and proposition for a classification. Hum Reprod. 2003;18(1):157-61.

5. Dai $Y$, et al. Clinico-pathologic characteristics of posterior deeply infiltrating endometriosis lesions, pain symptoms and its treatment using laparoscopic surgery. Zhonghua Fu Chan Ke Za Zhi. 2010;45(2):93-8.

6. Cao Q, et al. Comparison of complete and incomplete excision of deep infiltrating endometriosis. Int J Clin Exp Med. 2015;8(11):21497-506.

7. Somigliana $\mathrm{E}$, et al. Association rate between deep peritoneal endometriosis and other forms of the disease: pathogenetic implications. Hum Reprod. 2004;19(1):168-71.

8. Chapron C, et al. Associated ovarian endometrioma is a marker for greater severity of deeply infiltrating endometriosis. Fertil Steril. 2009;92(2):453-7.

9. Redwine DB. Ovarian endometriosis: a marker for more extensive pelvic and intestinal disease. Fertil Steril. 1999;72(2):310-5.

10. Chapron C, et al. Ovarian endometrioma: severe pelvic pain is associated with deeply infiltrating endometriosis. Hum Reprod. 2012;27(3):702-11.

11. Perello $\mathrm{M}$, et al. Markers of deep infiltrating endometriosis in patients with ovarian endometrioma: a predictive model. Eur J Obstet Gynecol Reprod Biol. 2017;209:55-60.

12. Kamergorodsky $\mathrm{G}$, et al. Histologic classification of specimens from women affected by superficial endometriosis, deeply infiltrating endometriosis, and ovarian endometriomas. Fertil Steril. 2009;92(6):2074-7.

13. Abbott JA, et al. The effects and effectiveness of laparoscopic excision of endometriosis: a prospective study with 2-5 year follow-up. Hum Reprod. 2003;18(9):1922-7

14. Bricou A, Batt RE, Chapron C. Peritoneal fluid flow influences anatomical distribution of endometriotic lesions: why Sampson seems to be right. Eur J Obstet Gynecol Reprod Biol. 2008;138(2):127-34.

15. Baessler K, Schuessler B. The depth of the pouch of Douglas in nulliparous and parous women without genital prolapse and in patients with genital prolapse. Am J Obstet Gynecol. 2000;182(3):540-4.

16. Sznurkowski J, Emerich J. Left lateral predisposition of endometrioma. Ginekol Pol. 2005;76(1):33-6.

17. Ruffo $G$, et al. Management of rectosigmoid obstruction due to severe bowel endometriosis. Updat Surg. 2014;66(1):59-64.

18. Nezhat C, et al. Urinary tract endometriosis treated by laparoscopy. Fertil Steril. 1996;66(6):920-4.

19. Kondo W, et al. Association between ovarian endometrioma and deep infiltrating endometriosis. Rev Bras Ginecol Obstet. 2012;34(9):420-4.

20. Razzaghi MR, Rahjoo T, Golshan A. Endometriosis with pure urinary symptoms. Urol J. 2009;6(2):132-4.

21. Oliveira MA, et al. Bowel complications in endometriosis surgery. Best Pract Res Clin Obstet Gynaecol. 2016;35:51-62.

22. Keckstein J, Wiesinger $\mathrm{H}$. Deep endometriosis, including intestinal involvement--the interdisciplinary approach. Minim Invasive Ther Allied Technol. 2005;14(3):160-6. 
23. Ruffo G, et al. Laparoscopic colorectal resection for deep infiltrating endometriosis: analysis of 436 cases. Surg Endosc. 2010;24(1):63-7.

24. Tarjanne S, et al. Complications and long-term follow-up on colorectal resections in the treatment of deep infiltrating endometriosis extending to bowel wall. Acta Obstet Gynecol Scand. 2015;94(1):72-9.

25. Zilberman S, et al. Partial Colpectomy is a risk factor for urologic complications of colorectal resection for endometriosis. J Minim Invasive Gynecol. 2013;20(1):49-55.

26. Gimonet $\mathrm{H}$, et al. Is pelvic MRI in women presenting with pelvic endometriosis suggestive of associated ileal, appendicular, or cecal involvement? Abdom Radiol (NY). 2016:41(12):2404-10.

27. Mabrouk $\mathrm{M}$, et al. Endometriosis of the appendix: when to predict and how to manage-a multivariate analysis of 1935 endometriosis cases. J Minim Invasive Gynecol. 2020;27(1):100-6.

28. Chopin N, et al. Relation between severity of dysmenorrhea and endometrioma. Acta Obstet Gynecol Scand. 2006;85(11):1375-80.

29. Dai Y, et al. Relationship of pelvic clinic-pathological features and the pain symptoms in ovarian endometrioma. Zhonghua Fu Chan Ke Za Zhi. 2013; 48(2):118-22.

30. Maccagnano C, et al. Ureteral endometriosis: proposal for a diagnostic and therapeutic algorithm with a review of the literature. Urol Int. 2013;91 (1):1-9.

31. Kolodziej A, et al. Urinary tract endometriosis. Urol J. 2015;12(4):2213-7.

32. Raimondo $D$, et al. Severe ureteral endometriosis: frequency and risk factors. J Obstet Gynaecol. 2018;38(2):257-60.

33. Suzuki T, et al. Impact of ovarian endometrioma on oocytes and pregnancy outcome in in vitro fertilization. Fertil Steril. 2005;83(4):908-13.

34. Vignali $\mathrm{M}$, et al. Surgical treatment of deep endometriosis and risk of recurrence. J Minim Invasive Gynecol. 2005;12(6):508-13.

35. Borghese $B$, et al. Recurrence of pain after surgery for deeply infiltrating endometriosis: How does it happen? How to manage? J Gynecol Obstet Biol Reprod (Paris). 2014;43(1):12-8.

36. Guo SW. Recurrence of endometriosis and its control. Hum Reprod Update. 2009;15(4):441-61.

37. Hachisuga T, Kawarabayashi T. Histopathological analysis of laparoscopically treated ovarian endometriotic cysts with special reference to loss of follicles. Hum Reprod. 2002;17(2):432-5.

38. Candiani M, et al. Ovarian recovery after laparoscopic enucleation of ovarian cysts: insights from echographic short-term postsurgical follow-up. J Minim Invasive Gynecol. 2005;12(5):409-14.

39. Ragni G, et al. Damage to ovarian reserve associated with laparoscopic excision of endometriomas: a quantitative rather than a qualitative injury. Am J Obstet Gynecol. 2005;193(6):1908-14.

\section{Publisher's Note}

Springer Nature remains neutral with regard to jurisdictional claims in published maps and institutional affiliations.

Ready to submit your research? Choose BMC and benefit from:

- fast, convenient online submission

- thorough peer review by experienced researchers in your field

- rapid publication on acceptance

- support for research data, including large and complex data types

- gold Open Access which fosters wider collaboration and increased citations

- maximum visibility for your research: over $100 \mathrm{M}$ website views per year

At $\mathrm{BMC}$, research is always in progress.

Learn more biomedcentral.com/submissions 